\title{
VELOCITY DIFFERENCE MEASUREMENT WITH A FIBER-OPTIC COUPLER
}

\author{
By \\ YIXUE DU \\ Bachelor of Science \\ Nanjing University \\ Nanjing, China
}

1994

Submitted to the Faculty of the

Graduate College of the Oklahoma State University in partial fulfillment of the requirements for the Degree of MASTER OF SCIENCE

December, 1998 


\section{VELOCITY DIFFERENCE MEASUREMENT WITH A FIBER-OPTIC COUPLER}

Thesis Approved:

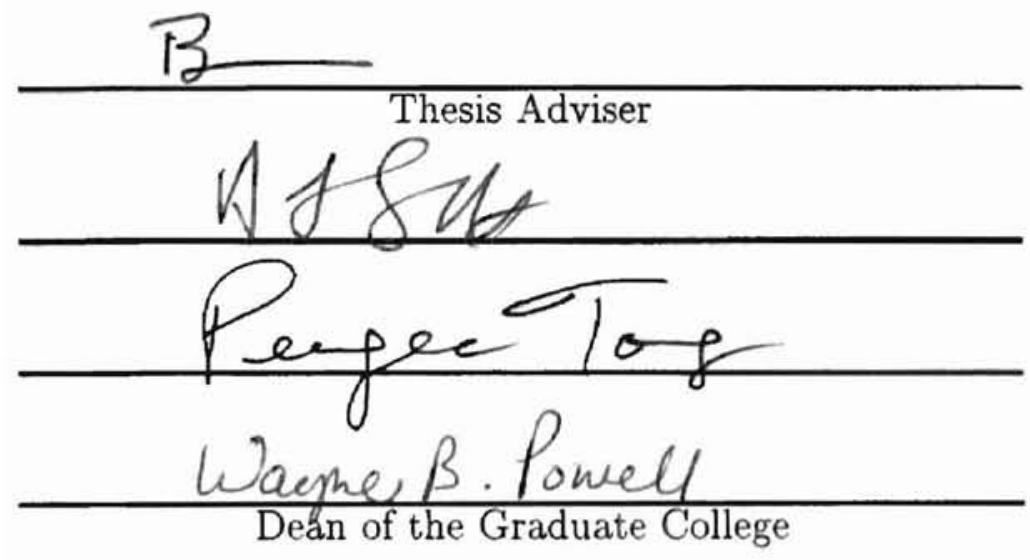




\section{ACKNOWLEDGMENTS}

This thesis would not have been possible without the help of all of my committee members. My adviser Dr. Bruce J. Ackerson's expert guidance and assistance was indespensible. Working in his lab has been a great learning experience for me. Dr. Penger Tong and Dr.Larry H. Scott kindly invested a great amount of time on my examination committee. For, this I am extremely grateful. I also appreciate all the discussions I have had with Dr. Tong which expanded my my views on the subject of light scattering. In some way the entire physics department contributed to this work. Without the assistance of Dr. Paul Westhaus with my visa troubles I would not have been able to come to Oklahoma State. Time and trees would not permit me to thank the countless teachers and colleagues who have assisted me in the past. This accomplishment I share with my parents and family who have given me constant support in this endeavor. Especially my sister Yibing, also being a colleague, she was the first one I reach for help when time came for the alignment of my experiment. For this I am eternally grateful. 


\section{TABLE OF CONTENTS}

Chapter

Page

I. INTRODUCTION . . . . . . . . . . . . . . 1

II. THEORY . . . . . . . . . . . . . . . . . . 4

III. EXPERIMENTS . . . . . . . . . . . . . . . . . . 9

IV. RESULTS AND DISCUSSION . . . . . . . . . . . . 13

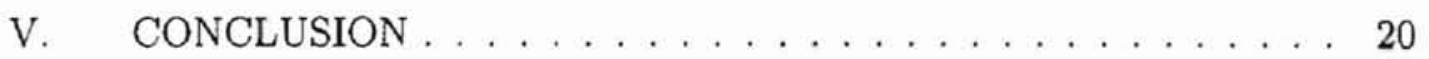

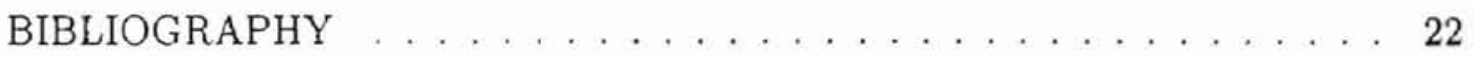




\section{LIST OF FIGURES}

Figure Page

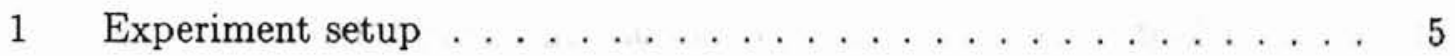

2 Intensity Profile . . . . . . . . . . . . . . . . . . 12

3 Intensity Autocorrelation Function . . . . . . . . . . . . . . 15

4 Oscillation Frequency vs. Separation . . . . . . . . . . . . . . 16

5 Correlation Function For Large Separation . . . . . . . . . . . . . . . 18 


\section{CHAPTER I}

\section{INTRODUCTION}

Dynamic light scattering has proven to be a powerful tool in the study of many dynamic processes. It has been used to probe fluctuations in systems at thermal equilibrium[1] and also those in non-equilibrium steady states, such as hydrodynamic fluctuations in laminar[2,3] and turbulent[4-7] flows. In the study of turbulent and other self-similar flows, one is interested in measuring the velocity difference, $\delta \mathbf{v}(\mathbf{r})=\mathbf{v}(\mathbf{x})-\mathbf{v}(\mathbf{x}+\mathbf{r})$, over varying distances $\mathbf{r}$ in a flow field $\mathbf{v}(\mathbf{x})$. As was demonstrated many years ago, $[8-10] \delta \mathbf{v}(\mathbf{r})$ is accessible by homodyne correlation spectroscopy (HCS). With the HCS scheme, small seed particles in a flowing fluid are used to scatter incident laser light. The scattered light intensity $I(t)$. which fluctuates because of the motion of the seed particles, contains beating frequencies of all particle pairs in the scattering volume. For each particle pair separated by a distance $\mathrm{r}$ (along the beam propagation direction), their Doppler beating frequency is $\mathbf{q} \cdot \delta \mathbf{v}(r)$, where $\mathbf{q}$ is the momentum transfer vector. The magnitude of $\mathbf{q}$ is given by $q=(4 \pi n / \lambda) \sin (\theta / 2)$, where $\theta$ is the scattering angle, $n$ is the refractive index of the fluid, and $\lambda$ is the wavelength of the incident light. Experimentally, the Doppler beating frequency is measured by the intensity auto-correlation function[1]

$$
g(\tau)=\frac{\langle I(t+\tau) I(t)\rangle}{\langle I(t)\rangle^{2}}=1+b G(\tau),
$$

where $b$ is an instrumental constant, and the angle brackets represent a time average over $t$. It has been shown that $G(\tau)$ in Eq. (1) has the form [4] 


$$
G(\tau)=\int_{0}^{L} d r h(r) \int_{-\infty}^{+\infty} d \delta v P(\delta v, r) \cos (q \delta v \tau),
$$

where $\delta v$ is the component of $\delta \mathbf{v}$ in the direction of $\mathbf{q}, P(\delta v, r)$ is the probability density function (PDF) of $\delta v(r)$, and $h(r) d r$ is the number fraction of particle pairs in the scattering volume. The weighted average over $r$ is required because the photo-detector receives light from particle pairs having a range of separations $(0<r<L)$. The function $G(\tau)$ yields information about the velocity differences in the direction of $\mathbf{q}$ and at various scales $r$ up to $L$. With the HCS technique, one measures the velocity differences directly without invoking Taylor's "frozen turbulence" assumption[11] to interpret the measurements.

In many previous experiments,[4-7] the length $L$ of the scattering volume viewed by a photo-detector was varied by changing the width $S$ of a slit in the collecting optics. With this "single slit" arrangement, the range of $L$ which could be varied in the experiment was rather limited. The lower cut-off for $L$ is controlled by the incident beam radius $\sigma$. In deriving Eq. (2), $L$ has been assumed to be much larger than $\sigma$, so that the integration over the beam radius can be neglected. When $L$ becomes smaller than $\sigma$, the decay of $g(\tau)$ is dominated by the velocity difference over $\sigma$ rather than $L$, and therefore Eq. (2) is no longer valid. The upper cut-off for $L$ is determined by the "coherence distance" (or coherence area) at the detecting surface of the photo-detector, over which the scattered electric fields are strongly correlated in space.[1] When the slit width $S$ becomes too large, the photo-detector sees many temporally fluctuating "speckles" (or coherence areas), and consequently fluctuations in the scattered intensity $I(t)$ will be averaged out over a range of $q$ values $\left(=q_{0} \pm \delta q\right)$ spanned by the detecting area. For spatially uncorrelated particle motions, such as Brownian diffusion, this spatial averaging over $\delta q$ only affects the signal-to-noise ratio of the measured $g(\tau)$, but the decay rate of $g(\tau)$ remains unchanged.[1] In turbulent and other self-similar flows, however, particle motions at different spatial points are strongly correlated and the spatial averaging effect changes both the signal-to-noise ratio and the functional form of $g(\tau) \cdot[2,12,13]$ 
In this paper we present a new "double slit" arrangement for the HCS technique, in which the Doppler shifted light from two different scattering regions separated by a distance $L$ is mixed on the photocathode. Experimentally, this mixing is accomplished by using a fiber-optic coupler, which couples two singlemode polarization preserving fibers. With the "double slit" arrangement, one can remove the upper limit for $L$ based on coherence areas and extend it to the coherence length of the laser to measure the velocity difference $\delta v(L)$ directly for any value of $L(>\sigma)$. In the study of turbulent flows, it is always desirable to expand the length scale range so that the scaling laws of $\delta v(L)$ over varying $L$ can be tested with high accuracy.[14] Furthermore, because fluctuations of the scattered intensity in the "double slit" arrangement are dominated by the Doppler beating frequency $\mathbf{q} \cdot \delta \mathbf{v}(L)$, the weighted average over $r$ in Eq. (2) is no longer needed. Therefore, the measured $G(\tau)$ becomes directly proportional to the Fourier cosine transform of the velocity $\operatorname{PDF} P(\delta v, L)$, if the single detector collection areas are sufficiently small.

The next section of this paper contains the theoretical calculation of the intensity auto-correlation function $g(\tau)$ in the "double slit" arrangement. Experimental details appear in Sec. 3 and the results are presented and analyzed in Sec. 4. Finally, the work is summarized in Sec. 5 . 


\section{CHAPTER II}

\section{THEORY}

Figure 1 shows the scattering geometry and the experimental setup. The two input optical fibers detect the scattered light from two different regions and then mix them at the coupler. The electric fields detected by each fiber sum in the coupler and consequently interfere. The intensity auto-correlation function $g(\tau)$ is then written as:

$g(\tau)=\frac{\left\langle\left[E_{1}(t+\tau)+E_{2}(t+\tau)\right]\left[E_{1}(t+\tau)+E_{2}(t+\tau)\right]^{*}\left[E_{1}(t)+E_{2}(t)\right]\left[E_{1}(t)+E_{2}(t)\right]^{*}\right\rangle}{\left\langle\left[E_{1}(t)+E_{2}(t)\right]\left[E_{1}(t)+E_{2}(t)\right]^{*}\right\rangle^{2}}$,

where $E_{1}$ and $E_{2}$ represent the electrical fields viewed by each of the input fibers with the polarization directions assumed to be the same. The numerator on the right hand side of the above equation contains 16 terms.[15] Eight of these terms are of the form $\left\langle E_{1} E_{2} E_{2} E_{2}\right\rangle$ or $\left\langle E_{2} E_{1} E_{1} E_{1}\right\rangle$ with one field contributed from one scattering volume and three from the other. Because the particles which scatter the light are assumed to be independently positioned in the scattering volumes 1 and 2, the averaging over each volume maybe carried out independently to obtain $\left\langle E_{1}\right\rangle\left\langle E_{2} E_{2} E_{2}\right\rangle$. Since a single field average $\left\langle E_{1}\right\rangle$ is zero, all these eight terms may be neglected. There are two more zero-value terms of the form $\left\langle E_{1}^{*}(t+\right.$ $\left.\tau) E_{1}^{*}(t) E_{2}(t+\tau) E_{2}(t)\right\rangle$ and $\left\langle E_{1}(t+\tau) E_{1}(t) E_{2}^{*}(t+\tau) E_{2}^{*}(t)\right\rangle$. These terms also separate into independent averages for each scattering volume and the averages for each scattering volume are zero for the same reason that $\left\langle E_{1}\right\rangle$ is. Two other terms are time independent and are equal to $2 I_{1} I_{2}$. Two of the remaining four terms are self-beating terms within each of the scattering volumes, $\left\langle I_{1}(t+\tau) I_{1}(t)\right\rangle$ and $\left\langle I_{2}(t+\right.$ $\left.\tau) I_{2}(t)\right\rangle$. The last two terms are conjugated cross-beating terms, which contain all the velocity difference information. When the velocity differences dominate 

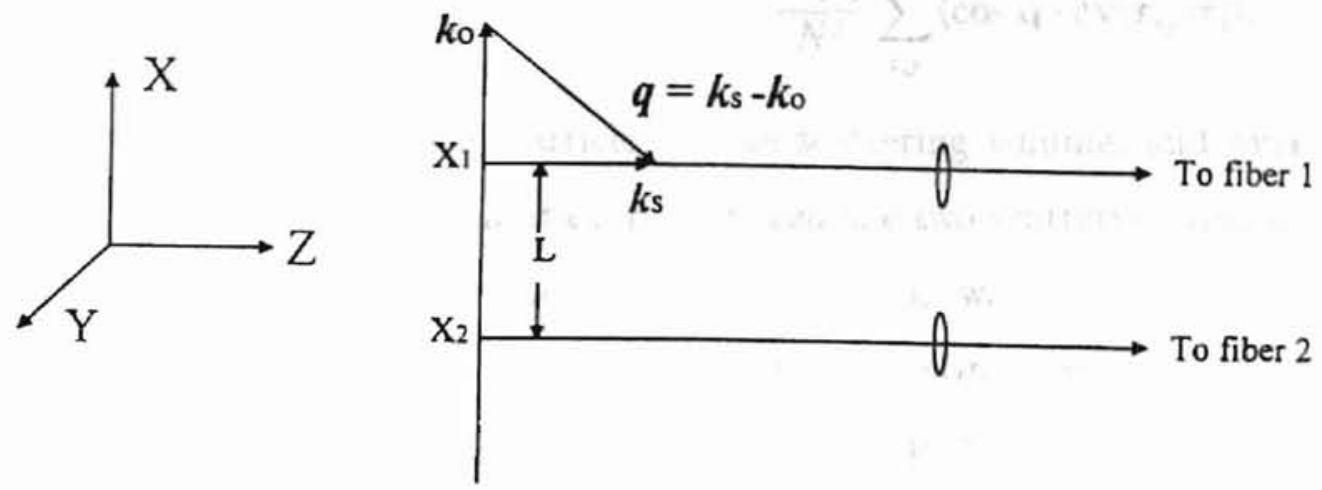

Incident beam

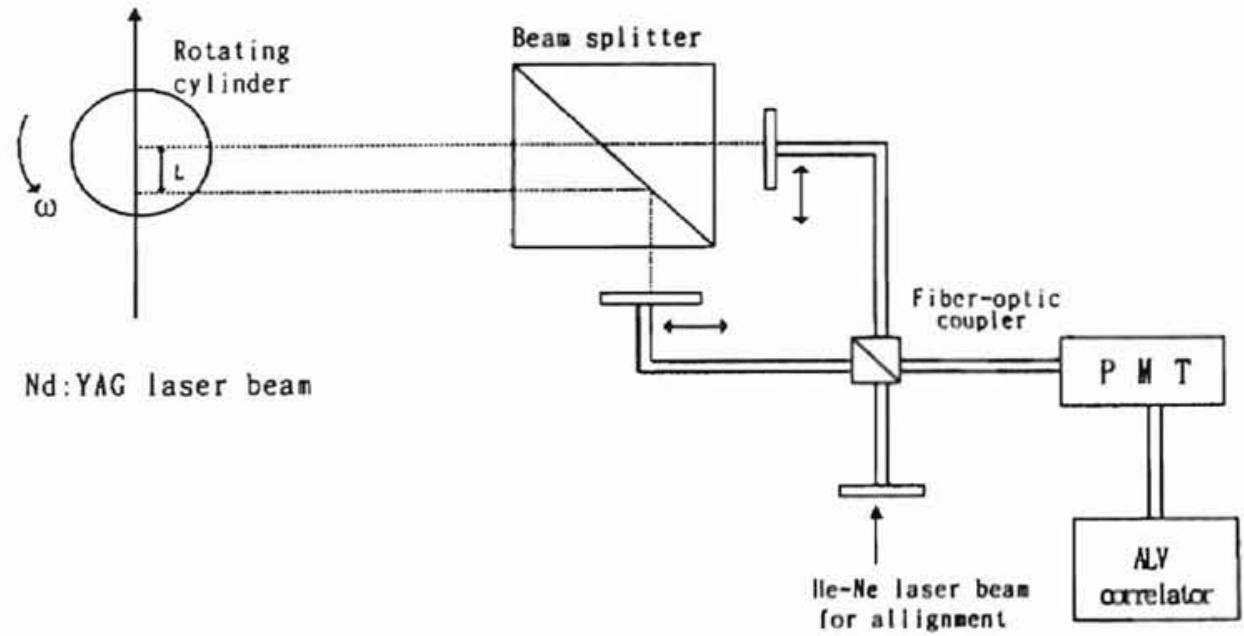

Figure 1. Schematic diagram of (a) the scattering geometry and (b) the experimental setup. 
intensity fluctuations, the cross-beating terms take the form[4]

$$
\left\langle E_{1}^{*}(t+\tau) E_{2}(t+\tau) E_{2}^{*}(t) E_{1}(t)\right\rangle+c . c=\frac{2 I_{1} I_{2}}{N^{2}} \sum_{i, j}^{N}\left\langle\cos \left[\mathbf{q} \cdot \delta \mathbf{v}\left(\mathbf{r}_{i j}\right) \tau\right]\right\rangle,
$$

where $N$ is the number of the particles in the scattering volume, and $\delta \mathbf{v}\left(\mathbf{r}_{i j}\right)$ is the velocity difference at a separation $\mathbf{r}_{i j}$ between the two scattering volumes. In deriving Eq. (4), we have assumed that the scattering wave vectors of the two electric fields are the same. If they have different scattering wave vectors, say $\mathbf{q}_{\mathbf{1}}$ and $\mathbf{q}_{2}$, the beating frequency in Eq. (4) will become $\mathbf{q}_{1} \cdot \mathbf{v}\left(\mathbf{r}_{i}\right)-\mathbf{q}_{2} \cdot \mathbf{v}\left(\mathbf{r}_{j}\right)$ instead of $\mathbf{q} \cdot \delta \mathbf{v}\left(\mathbf{r}_{i j}\right)$.

Equation (3) then becomes

$$
g(\tau)=1+\frac{I_{1}^{2}+I_{2}^{2}}{\left(I_{1}+I_{2}\right)^{2}} G_{s}(\tau)+\frac{2 I_{1} I_{2}}{\left(I_{1}+I_{2}\right)^{2}} G_{c}(\tau)=1+b_{s} G_{s}(\tau)+b_{c} G_{c}(\tau)
$$

In the above, the cross-beating correlation function is

$$
G_{c}(\tau)=\frac{1}{N^{2}} \sum_{i, j}^{N}\left\langle\cos \left[\mathbf{q} \cdot \delta \mathbf{v}\left(\mathbf{r}_{i j}\right) \tau\right]\right\rangle
$$

and the self-beating correlation function is

$$
G_{s}(\tau)=\frac{1}{N^{2}} \sum_{i, j}^{N}\left\langle\cos \left[\mathbf{q} \cdot \delta \mathbf{v}\left(\xi_{i j}\right) \tau\right]\right\rangle
$$

where $\delta \mathbf{v}\left(\xi_{i j}\right)$ is the velocity difference for a separation $\xi_{i j}$ within a single scattering volume 1 or 2 . For a homogeneous turbulent flow with a velocity $\operatorname{PDF} P(\delta \mathbf{v})$, we have

$$
\begin{aligned}
& G_{c}(\tau)=\iint_{-\infty}^{+\infty} d \mathbf{x} d \mathbf{x}^{\prime} f\left(\mathbf{x}, \mathbf{x}_{\mathbf{1}}\right) f\left(\mathbf{x}^{\prime}, \mathbf{x}_{\mathbf{2}}\right) \int_{-\infty}^{+\infty} d \delta \mathbf{v} P(\delta \mathbf{v}) \cos \left[\mathbf{q} \cdot\left(\mathbf{v}(\mathbf{x})-\mathbf{v}\left(\mathbf{x}^{\prime}\right)\right) \tau\right] \\
& =\int_{-\infty}^{+\infty} d \mathbf{r} h\left(\mathbf{r}, \mathbf{x}_{\mathbf{1}}, \mathbf{x}_{\mathbf{2}}\right) \int_{-\infty}^{+\infty} d \delta \mathbf{v} P(\delta \mathbf{v}) \cos [\mathbf{q} \cdot \delta \mathbf{v}(\mathbf{r}) \tau]
\end{aligned}
$$

where the number density of the particle pairs separated by a distance $\mathbf{r}$ is given by

$$
h\left(\mathbf{r}, \mathbf{x}_{1}, \mathbf{x}_{2}\right)=\int_{-\infty}^{+\infty} d \mathbf{x}^{\prime} f\left(\mathbf{r}+\mathbf{x}^{\prime}, \mathbf{x}_{1}\right) f\left(\mathbf{x}^{\prime}, \mathbf{x}_{2}\right) .
$$

In the above, the function $f\left(\mathbf{x}, \mathbf{x}_{1}\right)$ describes the intensity distribution of the scattering volume 1 viewed by an input optical fiber. The center of the scattering 
volume $\mathbf{1}$ is assumed to be at $\mathbf{x}_{\mathbf{1}}$. Equation (8) states that the light scattered by each pair of particles contributes a phase factor $\cos [\mathbf{q} \cdot \delta \mathbf{v}(\mathbf{r}) \tau]$ (because of the frequency beating) to the cross-beating correlation function $G_{c}(\tau)$, and $G_{c}(\tau)$ is an incoherent sum of these ensemble averaged phase factors over all the particle pairs across the two scattering volumes. With Eq. (8) one also can obtain the self-beating correlation function $G_{s}(\tau)$ by simply letting $\mathbf{x}_{1}=\mathbf{x}_{2}$. In this case, Eq. (8) becomes identical to Eq. (2).

In a typical light scattering experiment, the scattering volume viewed by an optical fiber can be assumed to be quasi one dimensional with its effective length $\ell_{0}$ being larger than the beam radius $\sigma$. In this case, the integration over $\sigma$ in Eq. (8) can be neglected. In fact, for the rigid body rotation to be described below, the integration over $\sigma$ is not needed, because the velocity gradient transverse to the beam direction is zero. Furthermore, it has been shown[16] that $f\left(\mathbf{x}, \mathbf{x}_{\mathbf{1}}\right)$ can be well approximated by a Gaussian function

$$
f\left(\mathbf{x}, \mathbf{x}_{1}\right)=\frac{1}{\sqrt{2 \pi \ell_{0}^{2}}} \exp \left[-\frac{\left(x-x_{1}\right)^{2}}{\ell_{0}^{2}}\right]
$$

where $\ell_{0}$ is the effective length of the scattering volume centered at $x_{1}$, and $x$ is the position variable along the incident laser beam [see Fig. 1(a)]. With Eq. (10) the function $h\left(\mathbf{r}, \mathbf{x}_{\mathbf{1}}, \mathbf{x}_{\mathbf{2}}\right)$ in Eq. (9) becomes

$$
h\left(\mathbf{r}, \mathbf{x}_{1}, \mathbf{x}_{2}\right)=\frac{0.2}{\ell_{0}} \exp \left[-\frac{\left(r+x_{2}-x_{1}\right)^{2}}{2 \ell_{0}^{2}}\right] .
$$

It can be shown using Fig. 1 that the beat frequency for rigid body rotation has the form $\mathbf{q} \cdot \delta \mathbf{v}\left(r_{i j}\right)=\left(\mathbf{k}_{\theta}-\mathbf{k}_{0}\right) \cdot \omega r_{i j} \mathbf{e}_{\theta}=k_{\theta} \omega(\sin \theta) r_{i j}$, where $k_{s}=k_{0}=2 \pi n / \lambda$. In the experiment to be described below, the scattering angle $\theta=90^{\circ}$. The function $G_{c}(\tau)$ in Eq. (8) then becomes

$$
\begin{aligned}
G_{c}(\tau) & =\frac{1}{2 \pi \ell_{0}^{2}} \iint_{-\infty}^{\infty} d x d x^{\prime} \exp \left[-\frac{\left(x-x_{1}\right)^{2}+\left(x^{\prime}-x_{2}\right)^{2}}{\ell_{0}^{2}}\right] \cos \left[k_{8} \omega\left(x-x^{\prime}\right) \tau\right] \\
& =\cos \left(a_{2} \tau\right) \exp \left[-\left(a_{1} \tau\right)^{2}\right],
\end{aligned}
$$

where $a_{1}=k_{s} \omega \ell_{0} / \sqrt{2}$ and $a_{2}=k_{s} \omega\left(x_{1}-x_{2}\right)=k_{s} \omega L$. In obtaining Eq. (12) we have omitted the integral over $\delta \mathbf{v}$ in Eq. (8), because $\delta \mathbf{v}$ is no longer a random 
variable for rigid body rotation. Similarly, one can obtain the function $G_{s}(\tau)$ by letting $x_{1}=x_{2}($ or $L=0)$ in Eq. (12). Thus we have $G_{s}(\tau)=\exp \left[-\left(a_{1} \tau\right)^{2}\right]$. The total correlation function $g(\tau)$ in Eq. (5) then becomes

$$
g(\tau)=1+b_{s} \exp \left[-\left(a_{1} \tau\right)^{2}\right]+b_{c} \cos \left(a_{2} \tau\right) \exp \left[-\left(a_{1} \tau\right)^{2}\right]
$$




\section{CHAPTER III}

\section{EXPERIMENTS}

Figure 1 shows the experimental set-up and the optical arrangement. The sample cell is a cylindrical cuvette having an inner diameter of $2.45 \mathrm{~cm}$ and a height of $5 \mathrm{~cm}$. It is filled with 1,2-propylene glycol which has refractive index $n=1.434$ and shear viscosity $\eta=40.4 \mathrm{mPas}$ (at $25^{\circ} \mathrm{C}$ ). This viscosity is 40 times larger than that of water. A small amount of polystyrene latex spheres of diameter $0.1 \mu \mathrm{m}$ is seeded in 1.2-propylene glycol at a volume fraction $\phi=1.5 \times 10^{-4}$. The top of the filled cylindrical sample cuvette was mounted so the axis of symmetry is coaxial with the axis of rotation of a geared motor. The motor produces smooth rotation with a uniform angular velocity $\omega=2.5 \mathrm{rad} / \mathrm{s}$. The whole sample cell was immersed in a large square vat of size $9 \mathrm{~cm} \times 9 \mathrm{~cm}$, which was also filled with 1,2-propylene glycol. The index matching vat was used to reduce parasitic scattering effects. Because the densities of 1,2-propylene glycol $\left(=1.036 \mathrm{~g} / \mathrm{cm}^{3}\right)$ and the polystyrene particles $\left(=1.05 \mathrm{~g} / \mathrm{cm}^{3}\right)$ are closely matched, settling is negligible. Because the viscosity of 1,2-propylene glycol is large, Brownian motion of the particles is negligible, and the whole fluid sample is essentially undergoing rigid body rotation. As a result, fluctuations of the scattered light intensity were dominantly produced by the rotational motion of the particles.

An incident beam from a Nd:YAG laser with an operating power of 200 $\mathrm{mW}$ and a wavelength of $\lambda=532.0 \mathrm{~nm}$ is focused at the center of the sample cell by a lens. The scattered light from two different regions is collected by a polarization preserving fiber-optic coupler (purchased from OZ-Optics), which consists of two input fibers and two output fibers coupled together [see Fig. 1(b)]. The four fiber arms are made of single-mode polarization maintaining (PM) fibers. Light from the two input fibers is first collimated, and then sent through the combiner (a 
beamsplitting cube) to divide each beam into two with a splitting ratio of $50 / 50$. The resultant output beams are then focused onto the two output fibers. The four fibers and the beamsplitting cube are carefully aligned and mounted together such that their polarization directions remain fixed. This ensures that the light from the two input fibers are well mixed and then split evenly into two output fibers. The two input fibers, both having a graded index lens for a better collection of light, are aimed at two different scattering spots through a large beamsplitting cube of square dimension $7.6 \mathrm{~cm}$ to points along the incident beam in the sample. To facilitate the change of the distance $L$ between the two scattering spots, we 111ounted each input fiber onto a micrometer-controlled translation stage. With this arrangement the distance $L$ can be varied from 0 to $250 \mathrm{~mm}$ in steps of $0.01 \mathrm{~mm}$. One of the output fibers was connected to a photomultiplier (PMT). The output signal from the PMT was then fed to an ALV-5000 correlator, which computed the auto-correlation function $g(\tau)$ of the scattered light.

The other output fiber was connected to a He-Ne laser, which was used for the optical alignment. With the reversed He-Ne light coming out of the input fibers, we could directly observe the scattering volume viewed by each input fiber, and align the input fibers such that they collect the scattered light with the same scattering angle $\theta\left(=90^{\circ}\right)$ or same $\mathrm{q}$ value for any distance $L$. Each input fiber is mounted on a small stage, whose orientation can be adjusted by two thumb screws. We installed a beam stop in the PMT housing to block the light from the He-Ne laser, when it was on. The intersection between the output He-Ne beam from a fiber and the incident beam from the Nd:YAG laser defines the scattering volume viewed by that input fiber. To verify the Gaussian assumption in Eq. (10), we used the reversed $\mathrm{He}-\mathrm{Ne}$ laser and measured the transverse intensity profile $I\left(x, x_{1}\right)$ of the output light for one of the "input" fibers. Figure 2 shows the normalized intensity profile $f\left(x, x_{1}\right) \equiv I\left(x, x_{1}\right) / I_{0}$, where $I_{0}$ is the peak intensity at $x=x_{1}$. The solid curve shows the fit of Eq. (10) to the data with $\ell_{0}=0.83 \mathrm{~mm}$. In the fitting we introduced a constant baseline to account for the room light contribution 
and the background noise of the photo-detector. It is seen that Eq. (10) fits the data well, confirming that the Gaussian assumption for $f\left(x, x_{1}\right)$ is indeed valid. 


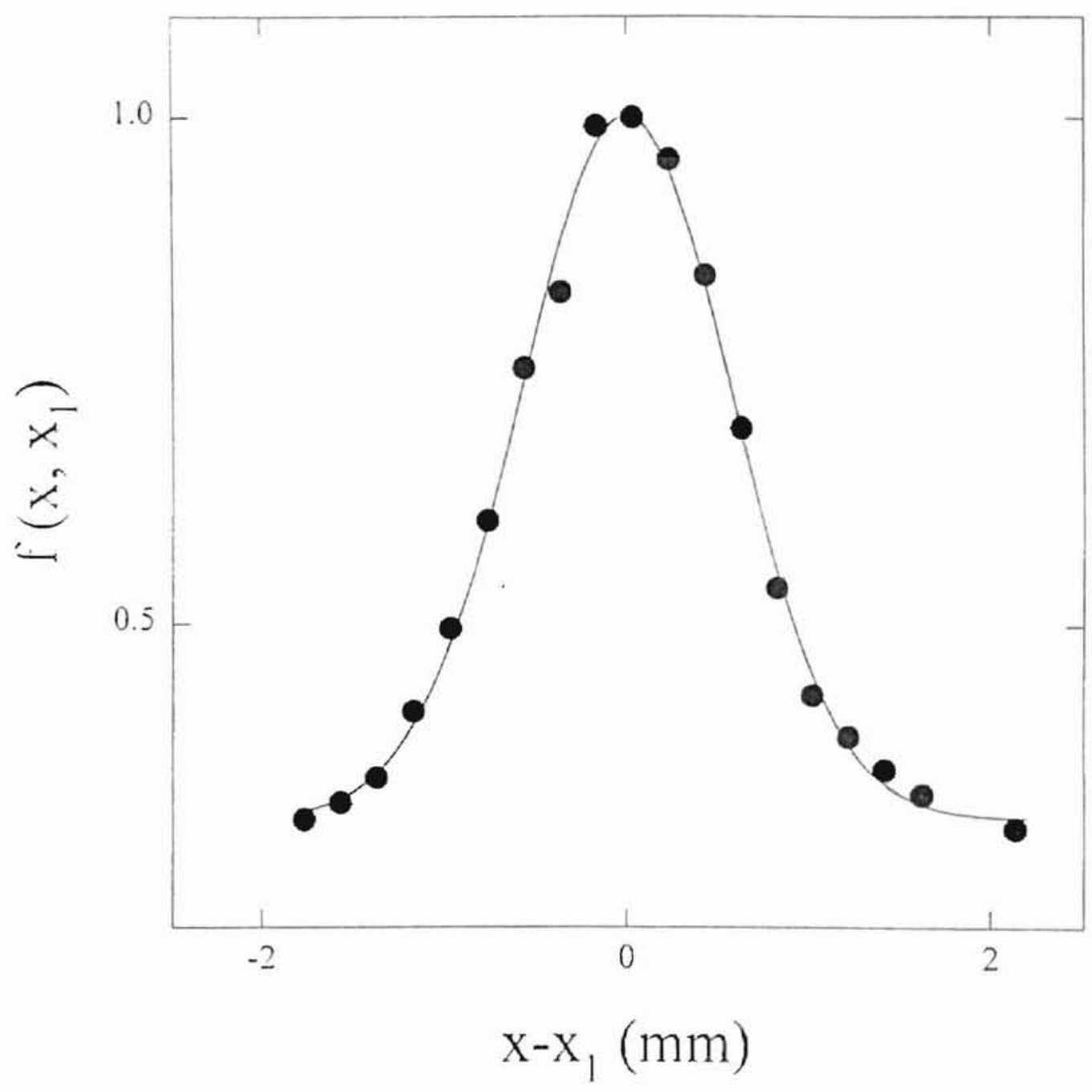

Figure 2. Measured intensity profile $f\left(x, x_{1}\right)$ as a function of $x-x_{1}$. The solid curve is a fit by Eq. (10) with $\ell_{0}=0.83 \mathrm{~mm}$. 


\section{CHAPTER IV}

\section{RESULTS AND DISCUSSION}

Figure 3(a) shows the measured $g(\tau)$ at $\omega=2.5 \mathrm{rad} / \mathrm{s}$ and $L=4.33 \mathrm{~mm}$ (circles). To show the oscillations in $g(\tau)$ more clearly, we plot the measured $g(\tau)$ with a log time scale. These oscillations reveal the characteristics of the frequency beating between the two input channels. The squares were obtained when one of the input optical fibers is blocked. In this case, the cross-beating correlation function $G_{c}(\tau)=0$, and therefore the measured $g(\tau)$ becomes $1+b_{s} G_{s}(\tau)$. Figure 3 (a) clearly shows that the squares represent an envelope for the circles. The lower solid curve in Fig.3(a) shows the fit of Eq. (13) to the circles with $a_{1}=$ $13.77(\mathrm{~ms})^{-1}, a_{2}=179.54(\mathrm{rad} / \mathrm{ms}), b_{s}=0.55$, and $b_{c}=0.332$. The upper solid curve is the fit of Eq. (13) to the squares with $G_{c}(\tau)=0, a_{1}=13.15(m s)^{-1}$, and $b_{s}=0.974$. It is seen that Eq. (13) fits the data well. Note that the fitted values of $a_{1}$ obtained from the two measurements are very close to one another.

From Eq. (12), one finds that the decay rate $a_{1}$ is proportional to the effective length $\ell_{0}$ of the scattering volume, and the oscillation frequency $a_{2}$ is proportional to the distance $L$ between the two scattering volumes. Therefore, the dynamic range for the measurement of the velocity difference $\delta v(L)$ is proportional to the length ratio $L / \ell_{0}$. To have a large dynamic range, one needs to reduce the value of $\ell_{0}$ as much as possible. For the experimental setup shown in Fig. 1(b), the value of $\ell_{0}$ is controlled by the graded index lens installed at the front end of each input fiber. The collimated beam diameter $(B D)$ is determined by $B D=2 f N A$, where $f$ is the focal length of the lens and $N A$ is the fiber numerical aperture. The full divergence angle $(D A)$, on the other hand, is determined by $D A=a / f$, where $a$ is the fiber core diameter. For the fiber-optic coupler used in the experiment, we have $f=3.2 \mathrm{~mm}, N A=0.1$, and $a=3.5 \mu \mathrm{m}$. Thus, we find $B D=0.64 \mathrm{~mm}$ and 
$D A=1.1 \mathrm{mrad}$. By changing the focal length $f$, one can minimize the length $\ell_{0}$ for a given distance between the input fiber and the scattering volume.

To further reduce the value of $\ell_{0}$, we added an additional lens in the collecting optics. Figure 3(b) shows the measured $g(\tau)$ obtained with the additional lens when $L=3.68 \mathrm{~mm}$ (circles). The squares were obtained when one of the input optical fibers was blocked. Because of the reduction of $\ell_{0}$, the decay rate $a_{1}$ becomes smaller. and therefore more oscillations appear in the measured $g(\tau)$. The upper solid curve in Fig. 3(b) shows the fit of Eq. (13) to the squares with $G_{c}(\tau)=0$, $a_{1}=3.71(\mathrm{~ms})^{-1}$, and $b_{s}=0.986$. The lower solid curve is the fit of Eq. (13) to the circles with $a_{2}=153.36(\mathrm{rad} / \mathrm{ms}), b_{s}=0.52$, and $b_{c}=0.41$. In the fitting, the value of $a_{1}$ was fixed at $3.71(\mathrm{~ms})^{-1}$. Using Eq. (12) we find $\ell_{0}=0.124 \mathrm{~mm}$ from the fitted value of $a_{1}=3.71(\mathrm{~ms})^{-1}$. In Fig. 2 we have shown the measurement of the intensity profile $I\left(x, x_{1}\right) / I_{0}$, from which we obtained a value of $\ell_{0}$. However, the measurement was carried out in air at a position outside the scattering sample. With a simple geometrical conversion, we find the actual value of $\ell_{0}=0.117 \mathrm{~mm}$ at the sample position inside the solution. This result compares well with the value of $l_{0}$ obtained from the fitted $a_{1}$.

We now discuss the $L$ dependence of the fitted oscillation frequency $a_{2}$. Figure 4 shows a $\log -\log$ plot of the fitted $a_{2}$ as a function of $L$. The data are found to be well described by a linear function $a_{2}=\alpha L$ with the slope $\alpha=$ $41.74 \mathrm{rad} / \mathrm{ms} / \mathrm{mm}$ (the solid line). According to Eq. (12), we find $a_{2}=\mathbf{q} \cdot \delta \mathbf{v}(L)=$ $k_{s} \omega L=42.32(\mathrm{rad} / \mathrm{ms} / \mathrm{mm}) L$, which is in good agreement with the measurement. It is seen in Fig. 4 that the value of $L$ spans over 2 orders of magnitude. As shown in Fig. 1(b), the upper limit for $L$ is controlled by either the sample size or the size of the large beam splitter. In fact, for large separations of the input fibers, the beam splitter is not needed and one can mount the fibers directly on a translation stage and measure the velocity difference $\delta v(L)$ at any value of $L$. Because of the large value of $L$, the measured $g(\tau)$ will contain many oscillations. Figure 5 shows the measured $g(\tau)$ at $L=9.68 \mathrm{~mm}$. The solid curve shows the fit of Eq. (13) to the data with $a_{1}=3.79(\mathrm{~ms})^{-1}, a_{2}=403.56(\mathrm{rad} / \mathrm{ms}), b_{s}=0.53$, 

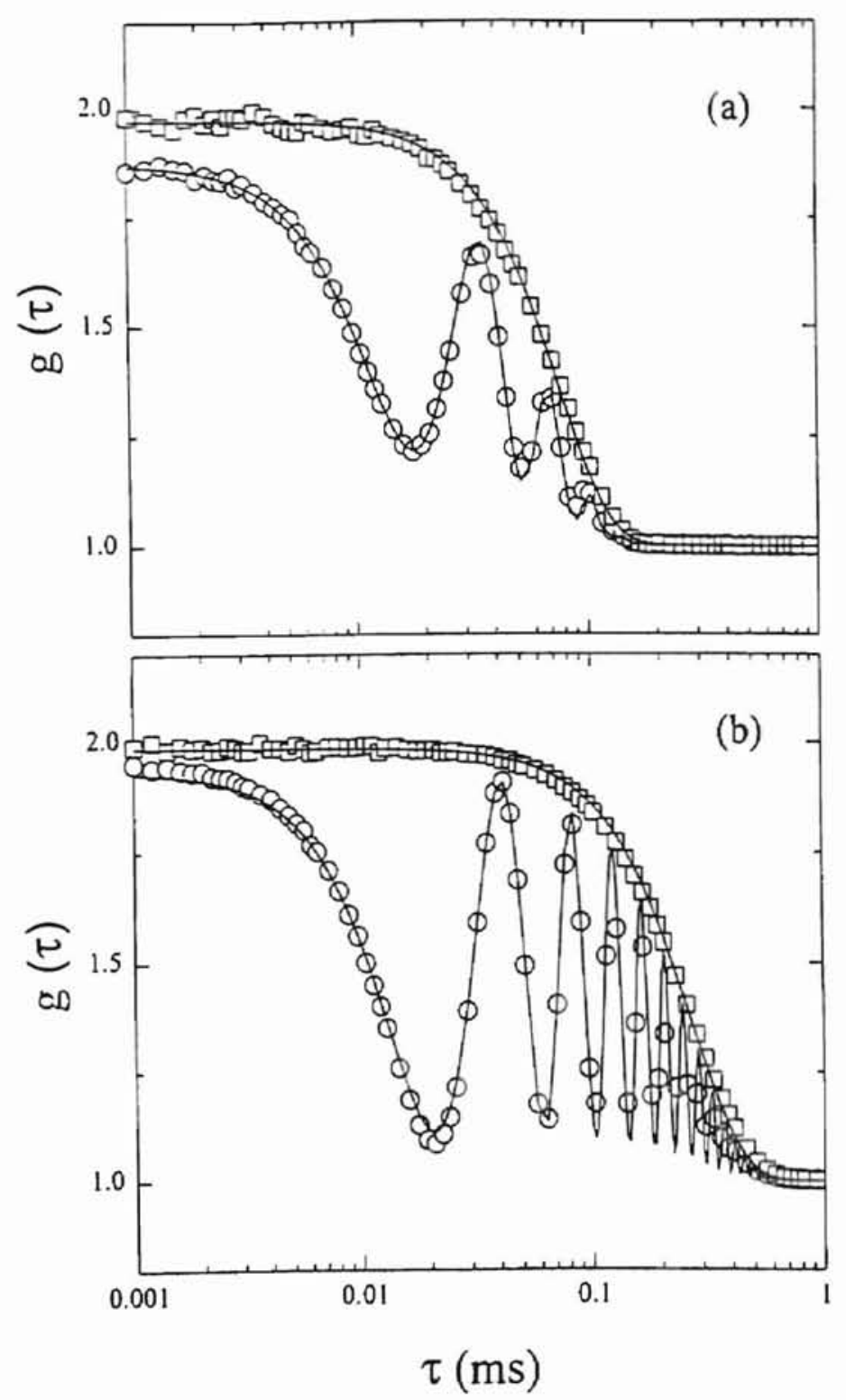

Figure 3. (a) Measured $g(\tau)$ as a function of the delay time $\tau$ when $\omega=2.5 \mathrm{rad} / \mathrm{s}$ and $L=4.33 \mathrm{~mm}$ (circles). The squares were obtained when one of the input optical fibers was blocked. (b) Measured $g(\tau)$ obtained with an additional lens when $L=3.68 \mathrm{~mm}$ (circles). The squares were obtained when one of the input optical fibers was blocked. The lower solid curves in (a) and (b) show the fits of Eq. (13) to the circles. The upper solid curves are the fits by Eq. (13) with $G_{c}(\tau)=0$. 


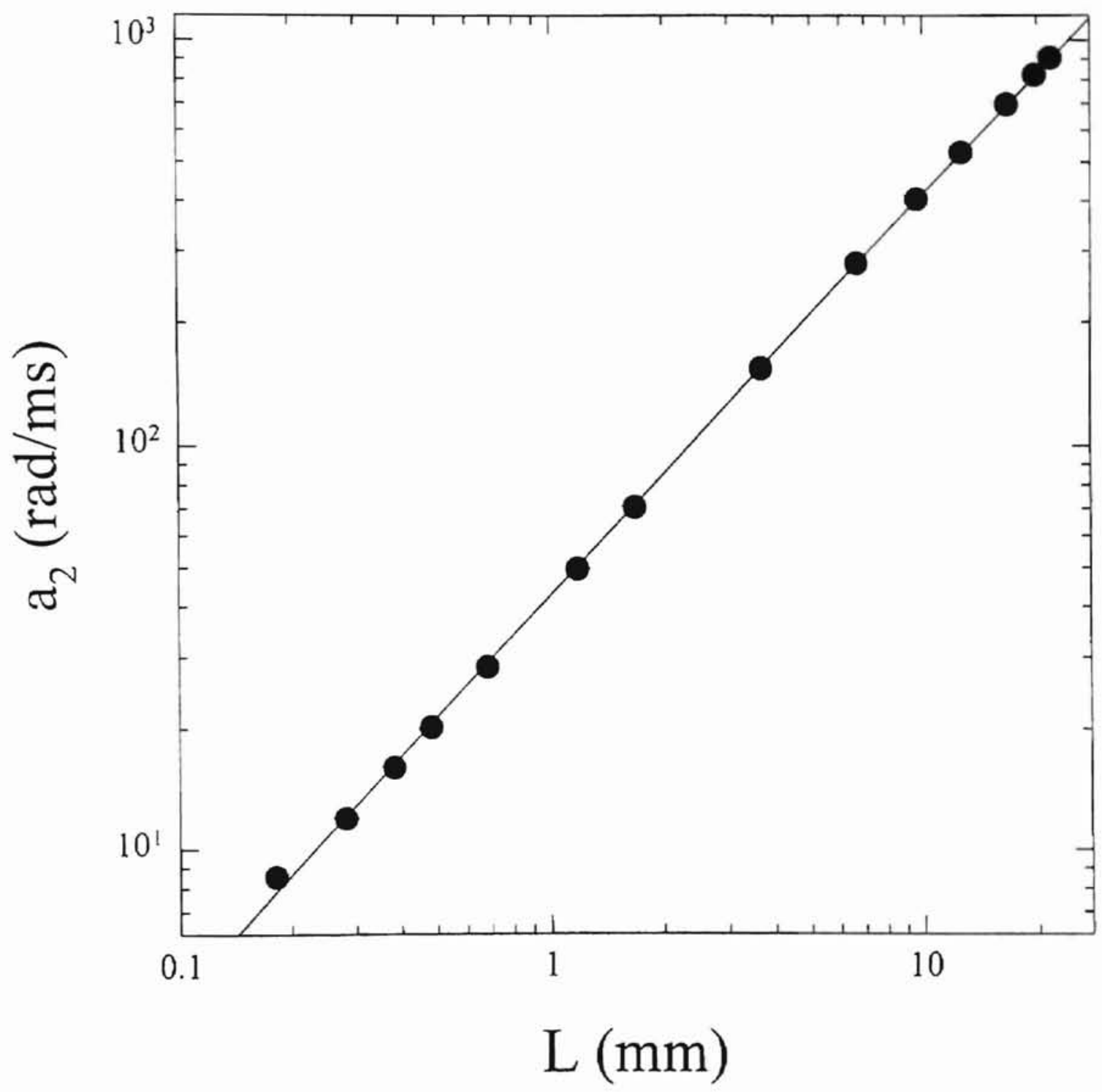

Figure 4. Log-log plot of the fitted $a_{2}$ as a function of $L$. The solid line shows the fitted linear function $a_{2}=41.74(\mathrm{rad} / \mathrm{ms} / \mathrm{mm}) L$. 
and $b_{c}=0.42$. It is seen that at large values of the delay time $\tau$, the measured $g(\tau)$ fails to follow the expected oscillations. This is because the delay channels of the ALV correlator used in the experiment are arranged logarithmically and the sampling time of each delay channel increases with the channel number. As a result, oscillations at large delay times are averaged out within each of the delay channels. Figure 5 thus suggests that a correlator with linear delay channels is best suited for the measurement of $\delta v(L)$ in a laminar flow. For turbulent flows, however, the velocity fluctuations will average out such oscillations in $g(\tau)$ and the Fourier cosine transform of the velocity $\operatorname{PDF} P(\delta v, L)$ [see Eq. (8)] will usually become a decaying function of $\tau$. In this case, a correlator with logarithmically spaced delay channels can be used to probe the long decay tail of the measured $g(\tau)$

In the experiment, the smallest value of $L$ can be adjusted to zero. In this case, the measured $g(\tau)$ should be identical to that obtained when one of the input optical fibers is blocked. It is found, however, that when the separation $L$ is set to zero, unusually large fluctuations appear in the measured scattering intensity. In fact, the intensity fluctuations were so large that we could not obtain any reliable $g(\tau)$. To find the origin of the intensity fluctuations at $L=0$, we turned on the reversed $\mathrm{He}-\mathrm{Ne}$ laser and let the two outgoing beams intersect at the sample position. The two beams interfered with each other at the crossing point and strong intensity fluctuations were observed visually. We believe that these intensity fluctuations are caused by small vibrations of the input fibers changing the optical path length. Once the separation $L$ becomes non-zero, the large intensity fluctuations disappear. As mentioned in Sec. 2, in deriving Eq. (12) we have omitted the integration over the beam radius $\sigma$. When $L$ becomes smaller than $\sigma$, the decay of $g(\tau)$ measured in a turbulent flow will be dominated by the velocity differences over $\sigma$ rather than $L$. Therefore, the lower limit of $L$ for the present technique is still determined by the radius of the incident laser beam.

Finally, we discuss the fitted values of $b_{s}$ and $b_{c}$ in Eq. (13). The sum of the two coefficients determines the value of $g(0)$ at $\tau=0$. In dynamic light 


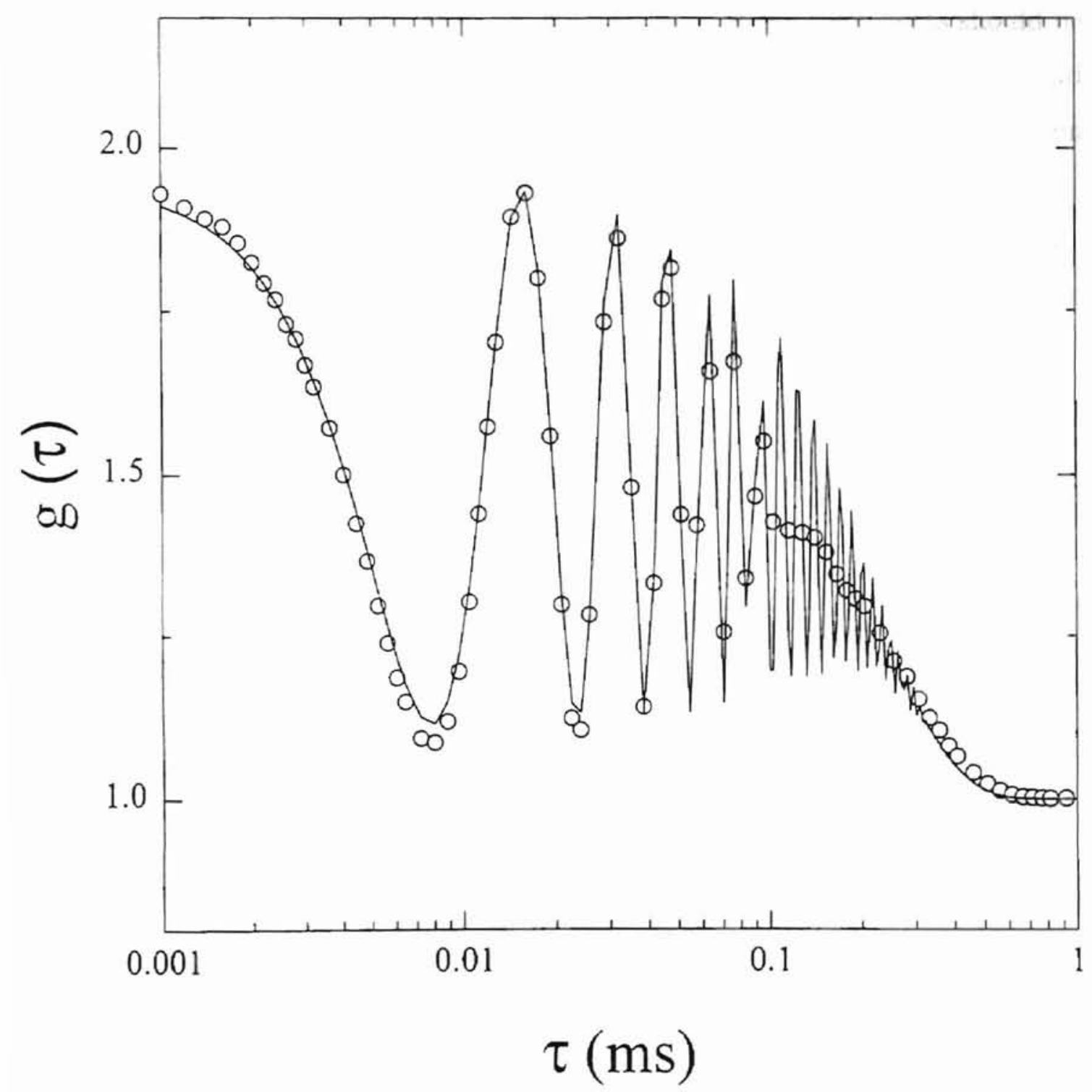

Figure 5. Measured $g(\tau)$ as a function of $\tau$ when $\omega=2.5 \mathrm{rad} / \mathrm{s}$ and $L=9.68 \mathrm{~mm}$. The solid curve is a fit by Eq. (13). 
scattering measurements, $g(0)$ is a measure of the signal-to-noise ratio for the correlation function $g(\tau)$. According to Eq. (5), both of the coefficients should be equal to 0.5 when $I_{1}=I_{2}$. From the fitting in Fig. 3(b), we have $b_{s}=0.52$ and $b_{c}=0.41$. It is found that the value of $b_{c}$ depends strongly on the polarization state of the electric fields in the two input fibers. To have a perfect mixing [i.e. $g(0)=2$, the polarization of the two electric fields needs to be exactly parallel. Prior to the purchase of the polarization preserving fiber-optic coupler, we used a non-polarization preserving fiber-optic coupler to measure $g(\tau)$. This coupler was made with non-polarization maintaining (non-PM) single-mode fibers. Because the polarization of the E-field inside a non-PM fiber changes with the spatial configuration of the fiber, the measured $g(\tau)$ is found to be very sensitive to the spatial arrangement of the input fibers. In many cases, the measured $g(0)$ is found to be $\sim 1.5$, indicating that the cross-beating term $G_{c}(\tau) \simeq 0$ [see Eq. (5)]. In fact, the measured $g(\tau)$ shown in Fig. 3(a) was obtained using the non-PM coupler under the "best fiber arrangement". Still, the measured $g(0)$ (circles) is found to be substantially lower than that for the self-beating term (squares) [see Fig. 3(a)]. When the polarization preserving fiber-optic coupler is used, the measured $g(0)$ becomes insensitive to the spatial arrangement of the input fibers. Furthermore, the measured $g(0)$ (circles) is found to be very close to that for the self-beating term (squares) [see Fig. 3(b)]. To study an unknown turbulent flow, it is important to use a polarization preserving fiber-optic coupler to measure the velocity difference $\delta v(L)$. In this way, one can ensure that the changes of the measured $g(\tau)$ are caused by the flow not by the optics. 


\section{CHAPTER V}

\section{CONCLUSION}

We have developed a new optical arrangement for photon correlation spectroscopy (PCS) to measure the velocity difference $\delta v(L)$ at a separation $L$. In the experiment, two single mode fibers are used to collect light with the same scattered wavevector but from two spatially separated regions in a sample. These regions are illuminated by a single coherent laser beam, so that the collected signals interfere when combined using a fiber optic coupler, before being directed to a photomultiplier tube. The fibers and coupler are polarization preserving to guarantee a high signal-to-noise ratio. We calculate the intensity correlation function $g(\tau)$ for this new "double slit" arrangement. Experiment verifies the theory in a simple case of rigid body rotation and demonstrates its applications. The measured $\delta v(L)$, which spans over two orders of magnitude in $\mathrm{L}$, agrees with the theoretical prediction with an error less than $5 \%$.

The new fiber-optic method has several advantages over the usual "single slit" arrangement for PCS. First, the use of the fibers and coupler makes the optical alignment much easier. With the unique design of the fiber-optic coupler, one is able to conduct on-line alignment and calibrations. Second, because fluctuations of the scattered light in the "double slit" arrangement is dominated by the interference of light from two different scattering regions with a separation $L$, information about $\delta \mathbf{v}(L)$ can be obtained directly from the measured $g(\tau)$ without introducing an additional integration. As a result, the "double slit" arrangement becomes more sensitive to the probability density function (PDF) of $\delta \mathbf{v}(L)$. Finally, with the new scheme one can remove the upper limit for $L$ based on coherence areas and extend 
it to the coherence length of the laser. Therefore, one can measure $\delta v(L)$ accurately over a much wider range of $L$. We expect that the new fiber-optic method is useful for the study of turbulent flow and particle settling dynamics. 


\section{BIBLIOGRAPHY}

1. B. J. Berne and R. Pecora, "Dynamic light scattering"(Wiley, New York, 1976).

2. B. J. Ackerson and N. A. Clark, "Dynamic light scattering at low rates of shear," J. de Physique, 42, 929-936 (1981).

3. G. G. Fuller, J. M. Rallison, R. L. Schmidt, and L. G. Leal, "The measurement of velocity gradients in a laminar flow by homodyne lightscattering spectroscopy,"J. Fluid Mech., 100, 555-575 (1980).

4. P. Tong, W. I. Goldburg, C. K. Chan, and A. Sirivat, "Turbulent transition by photon correlation spectroscopy," Phys. Rev. A, 37, 2125-2133 (1988).

5. H. K. Pak. W. I. Goldburg, and A. Sirivat, "Measuring the probability distribution of the relative velocities in grid-generated turbulence,"Phys. Rev. Lett., 68, 938-941 (1992); "An experimental study of weak turbulence,"Fluid Dyn. Res., 8, 19-31 (1991).

6. P. Tong and Y. Shen, "Relative velocity fluctuations in turbulent RayleighBénard convection," Phys. Rev. Lett. 69, 2066-2069 (1992).

7. H. Kellay, X.-1. Wu, and W. I. Goldburg, "Experiments with turbulent soap films,"Phys. Rev. Lett. 74, 3975-3978 (1995).

8. P. J. Bourke, J. Butterworth, L. E. Drain, P. A. Egelstaff, A. J. Hughes, P. Hutchinson, D. A. Jackson, E. Jakeman, B. Moss, J. O'Shaughnessy, E. R. Pike, and P. Schofield, "A study of the spatial structure of turbulent flow by intensity-fluctuation spectroscopy," J. Phys. A, 3, 216-228 (1970).

9. S. Hanson, "The laser gradient anemometer," in Photon Correlation Technıques in Flu2d Mechancs, p. 212-220, edited by E. O. Schulz-DuBois (Springer, Berlin, 1983).

10. C. Keveloh and W. Staude, "The application of cross-correlation measurements to the determination of velocity gradients in flui flow," in Photon Correlation Techniques in Fluid Mechanics, p. 221-224, edited by E. O. Schulz-DuBois (Springer, Berlin, 1983). 
11. G. I. Taylor, "The spectrum of turbulence,"Pro. R. Soc. London A, 164, 476-490 (1938).

12. K. J. Måløy, W. I. Goldburg, and H. K. Pak, "Spatial coherence of homodyne light scattering from particles in a convective field,"Phys. Rev. A 46, 3288-3291 (1992).

13. T. Narayanan, C. Cheung, P. Tong, W. I. Goldburg, and X.-L. Wu, "Measurement of the velocity difference by photon correlation spectroscopy: an improved scheme," Applied Optics, 36, 7639-7644 (1997).

14. see, e.g., P. Tong and W. I. Goldburg, "Relative velocity fluctuations in turbulent flows at moderate Reynolds number II. Model calculation," Phys. Fluids 31, 3253-3259 (1988).

15. H. Z. Cummins and H. L. Swinney, "Light beating spectroscopy," in Progress in optics VIII, p. 133-200, edited by E. Wolf (North-Holland, Amsterdam, 1970).

16. U. Nobbmann, S. W. Jones and B. J. Ackerson, "Multiple scattering suppression: cross-correlation with titled single-mode fibers," Applied Optics, 36, 7571-7582 (1997). 


\section{VITA \\ YIXUE DU \\ Candidate for the Degree of \\ Master of Science}

\section{Thesis: VELOCITY DIFFERENCE MEASUREMENT WITH A FIBER- OPTIC COUPLER}

Major Field: Physics

Biographical:

Personal Data: Born in Taiyuan/Shanxi, China, September 24, 1972, the daughter of Peizhi Huang and Yuemin Du.

Education: Graduated from Nankai Highschool, Tianjin, China, June, 1990; Recieved Bachelor of Science in Acoustics from Nanjing University, Nanjing, China, July, 1994; completed the requirements for the Master of Science Degree at the Oklahoma State University, Stillwater, Oklahoma, December, 1998. 